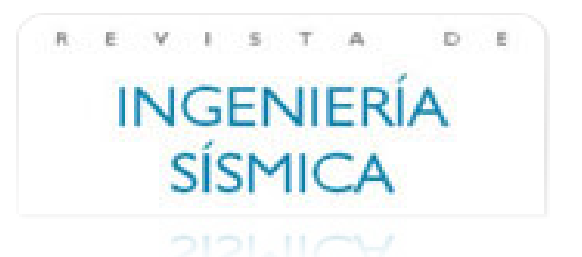

\section{Revista de Ingeniería Sísmica}

ISSN: 0185-092X

javiles@tlaloc.imta.mx

Sociedad Mexicana de Ingeniería Sísmica

México

De la Colina Martínez, Jaime

Respuesta torsional de edificios en el marco del análisis pushover

Revista de Ingeniería Sísmica, núm. 75, 2006, pp. 47-67

Sociedad Mexicana de Ingeniería Sísmica

Distrito Federal, México

Disponible en: http://www.redalyc.org/articulo.oa?id=61807503

- Cómo citar el artículo

Número completo

- Más información del artículo

Página de la revista en redalyc.org

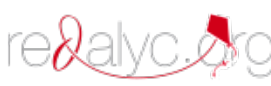

Sistema de Información Científica

Red de Revistas Científicas de América Latina, el Caribe, España y Portugal

Proyecto académico sin fines de lucro, desarrollado bajo la iniciativa de acceso abierto 


\title{
RESPUESTA TORSIONAL DE EDIFICIOS EN EL MARCO DEL ANÁLISIS PUSHOVER
}

\author{
Jaime De la Colina Martínez ${ }^{(1)}$
}

\begin{abstract}
RESUMEN
Este trabajo emplea el análisis pushover clásico para observar los efectos de excentricidad, fuerzas laterales ortogonales y de los factores de amplificación empleados en el diseño por torsión en la respuesta lateral estática no lineal de edificios, a base de marcos tridimensionales de baja altura. El objetivo de este estudio es identificar si con este análisis, se observan los mismos efectos y comportamientos de algunas de las principales variables dentro del problema de torsión que se han observado en análisis dinámicos no lineales previos. Los cálculos muestran que los resultados obtenidos a través del análisis pushover son consistentes con las conclusiones derivadas de análisis dinámicos tridimensionales no lineales. Esto sustenta la hipótesis de que el análisis pushover clásico puede ser una herramienta de mucho valor para el diseño y la evaluación de edificios tridimensionales con torsión a base de marcos.
\end{abstract}

\begin{abstract}
The classical pushover analysis is used to show the effects of eccentricity, orthogonal lateral forces, and torsion-design amplification factors on the nonlinear static lateral response of low-rise torsionally unbalanced (TU) frame buildings. The purpose of this study is to identify if this analysis captures the same effects of the main torsion variables that have been observed through previous dynamic nonlinear analysis. Computations show that pushover analysis results are consistent with the conclusions derived from three dimensional dynamic nonlinear analyses. This supports the hypothesis that pushover analysis can be a valuable tool for either design or evaluation of three dimensional frame buildings with twist.
\end{abstract}

\section{INTRODUCCIÓN}

Las nuevas tendencias en el diseño sísmico de edificios, parecen estar orientadas a la estimación del comportamiento estructural a diferentes niveles de la intensidad del movimiento del terreno ocasionado por sismos (BSSC, 1997). Para ello, el uso del análisis pushover ha sido identificado como una importante herramienta computacional. Algunas de las ventajas y desventajas de usar este tipo de análisis ya han sido discutidas anteriormente (Kim y D’Amore, 1999) pero, en general, parece ser una herramienta útil que incorpora la mayoría de las características del edificio relacionadas con la capacidad última en la evaluación de su resistencia lateral.

Artículo recibido el 3 de octubre de 2005 y aprobado para su publicación el 7 de abril de 2006. Se aceptarán comentarios y/o discusiones hasta cinco meses después de su publicación.

${ }^{1}$ Facultad de Ingeniería. Universidad Autónoma del Estado de México. Ciudad Universitaria/Cerro de Coatepec s/n. Toluca, Estado de México. jcolina@uaemex.mx 
Esta observación, sin embargo, se ha obtenido principalmente del estudio de estructuras planas, como se ha observado en recientes documentos (ATC, 2004).

Una limitación interesante del análisis pushover clásico es la distribución con la altura de las cargas laterales, la cual es invariante y que, en general, no toma en cuenta la contribución de los modos superiores de vibración. Por esta razón, se han propuesto algunas variantes del análisis pushover clásico u original. Por ejemplo, Bracci et al (1997) propusieron una distribución ajustable de cargas laterales con la idea de obtener mejores estimaciones de las demandas sísmicas. Asimismo, el análisis pushover modal propuesto recientemente (Chopra y Goel, 2002), constituye un procedimiento alternativo que pretende incorporar la contribución de los modos superiores de vibración. Otros procedimientos similares ya han sido propuestos previamente, como el de Paret et al (1996) y el de Sasaki et al (1998). En relación con estos últimos trabajos, es importante comentar, sin embargo, que con la búsqueda de procedimientos tipo pushover que proporcionen resultados más próximos a los que se pudieran obtener con una análisis no lineal dinámico, se está perdiendo una de las características principales del pushover clásico original; i.e., con los recientes métodos propuestos (e.g. pushover modal) se requiere de tantos pasos de cálculo que prácticamente deja de ser atractivo para el ingeniero, como lo ha señalado recientemente Maison (2005).

Por otro lado, aun cuando la mayoría de los análisis pushover se han aplicado a estructuras planas (2D), con el análisis pushover clásico también se pueden estudiar estructuras tridimensionales (3D) con la ayuda de programas pushover específicos (Kilar y Fajfar, 1997; Li, 1996). Algunos de estos programas incluso han sido útiles para la evaluación de nuevos procedimientos para evaluar el daño en edificios ocasionado por sismos, particularmente de aquellos con torsión (e.g., Mohadam y Tso, 1996).

Antes de extender el uso de los análisis tipo pushover a otras aplicaciones, parece importante evaluar el comportamiento de este método de análisis al caso de estructuras 3D con torsión. Es posible que el trabajo en esta dirección pueda conducir a considerar el análisis pushover como una herramienta importante (y confiable) para el diseño sísmico, incluyendo el de edificios torsionalmente desbalanceados (TD).

Resulta interesante también, por ejemplo, estudiar el efecto de las principales variables involucradas en el problema de torsión de edificios dentro del contexto del análisis pushover. Es importante tener en mente, antes de incorporar el análisis pushover en alguna metodología de diseño, que el ingeniero debe contar igualmente con procedimientos simples para estimar la capacidad lateral última de edificios.

En este artículo, se presentan resultados para aplicar el análisis pushover clásico a modelos de edificios a base de marcos de cinco niveles, con la idea de entender cómo este tipo de análisis muestra el efecto que tienen las principales variables para distintas respuestas estructurales. Ya que el análisis pushover no toma en cuenta las características dinámicas de la estructura, y debido al alcance inicial de este estudio, aquí se consideran las siguientes variables: 1) excentricidad estructural a lo largo de una dirección del modelo, 2) factores para el cálculo de excentricidades estáticas de diseño por torsión y 3) fuerzas laterales ortogonales. 
Las siguientes fórmulas son generalmente consideradas (ver por ejemplo De-la-Llera y Chopra, 1994) para calcular los momentos de torsión $\left(M_{T}=V_{b} \cdot e_{d}\right)$ que son combinados con el cortante basal $\left(V_{b}\right)$ para el diseño de los elementos resistentes a carga lateral

excentricidad primaria

excentricidad secundaria

$$
\begin{aligned}
& e_{d 1}=\alpha e_{s}+\beta b \\
& e_{d 2}=\delta e_{s}-\beta b
\end{aligned}
$$

donde $\alpha$ y $\delta$ son factores que modifican la excentricidad natural de entrepiso $e_{s}$ para tomar en cuenta el comportamiento dinámico (con un diseño estático). El factor $\alpha$, por ejemplo, amplifica las fuerzas calculadas estáticamente en los marcos donde la torsión es desfavorable. Esta amplificación se debe a que la respuesta dinámica conduce a fuerzas mayores en estos marcos que las fuerzas calculadas estáticamente. El factor $\delta$ indica la porción del cortante favorable que la torsión causa en algunos marcos y que puede restarse del cortante directo. Este factor también trata de compensar la diferencia entre los cortantes calculados estáticamente de aquellos calculados dinámicamente. Por ejemplo, si $\delta=0.5$, este factor indica que sólo debe descontarse el $50 \%$ del cortante favorable que la torsión causa en algunos marcos. El factor $\beta$ se relaciona con la excentricidad accidental. Este artículo se relaciona con los factores $\alpha$ y $\delta$ solamente. La excentricidad accidental no se modela en este trabajo, por lo que no se incluye el estudio de $\beta$ en esta investigación (i.e., $\beta=0$ ).

Cabe mencionar que cuando la excentricidad $e_{s}$ se debe a una distribución asimétrica de rigideces, el cálculo de $e_{s}$ resulta dependiente la distribución de cargas laterales (Alcocer, 1986).

Es importante aclarar que este trabajo no está orientado a evaluar la precisión con la cual el análisis pushover estima las demandas sísmicas, como lo hacen otros estudios similares (por ejemplo, en el caso del pushover modal ver Goel y Chopra, 2004). Por esta razón, no se intenta aquí comparar los resultados del análisis pushover con aquellos obtenidos de un análisis dinámico (no lineal). Sólo se usa la metodología del análisis pushover clásico para observar si éste es capaz de identificar los efectos de algunas de las variables involucradas en el problema de torsión de edificios que se han observado en estudios dinámicos similares realizados antes (e.g., De-laColina, 1999).

Esta investigación describe primeramente la geometría y las propiedades de los modelos de edificios considerados y, posteriormente, presenta el efecto de la excentricidad y de los factores de amplificación $\alpha$ y $\delta$ dentro del contexto del análisis pushover.

\section{MODELOS}

Se consideran modelos de edificios a base de marcos con tres marcos resistentes a carga lateral en cada dirección ortogonal. Como se ilustra en la figura 1, se estudian modelos con dos relaciones largo/ancho. El lado más grande de ambas plantas es igual a $16 \mathrm{~m}$ y la excentricidad natural $e_{s}$ (con $e_{s}=e \cdot b$ ) se mide a lo largo de este lado (por tanto, $b=16 \mathrm{~m}$ en ambos casos). No se considera excentricidad en la dirección ortogonal. La tabla 1 contiene la información de las propiedades de las secciones transversales de vigas y columnas. Como usualmente se hace en la práctica, estas propiedades han sido agrupadas en tres conjuntos por conveniencia en el diseño. 
Se puede observar que las columnas se han seleccionado con secciones transversales cuadradas; por lo que, de acuerdo con Humar y Kumar (1999), esta selección conduce a diferentes valores del cociente de rigidez torsional $\gamma$ para ambas plantas $(\gamma=$ cociente de rigidez torsional que aportan los planos paralelos al movimiento del terreno entre la rigidez torsional total del edificio). Dichos autores definieron este cociente para sistemas de un piso con vigas rígidas a flexión, donde la rigidez lateral sólo depende de los elementos verticales. Aun cuando las vigas también contribuyen a la rigidez torsional, aquí el cociente $\gamma$ se calculó asumiendo las vigas como infinitamente rígidas a flexión.

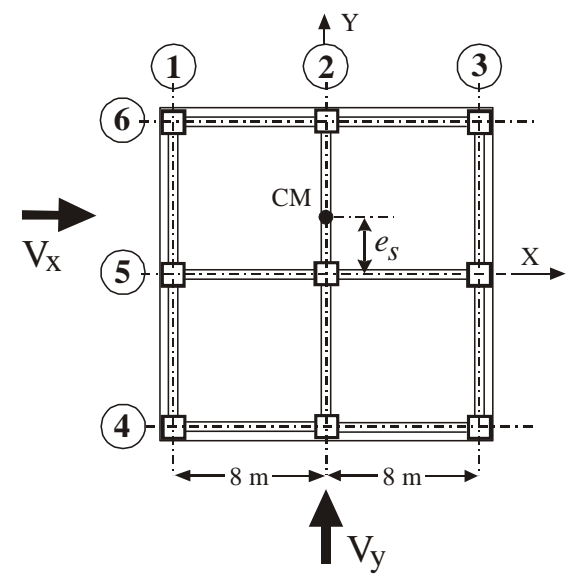

a) Planta cuadrada

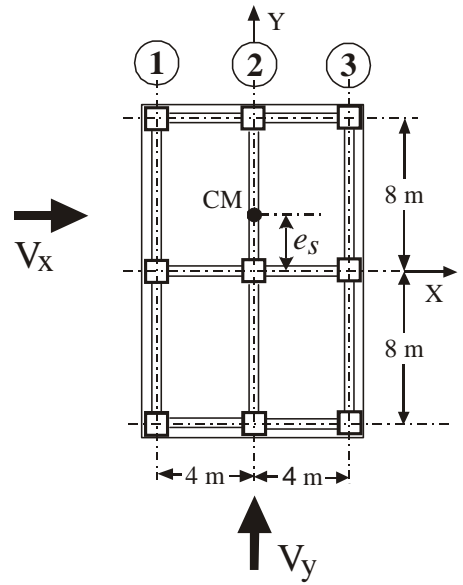

b) Planta rectangular

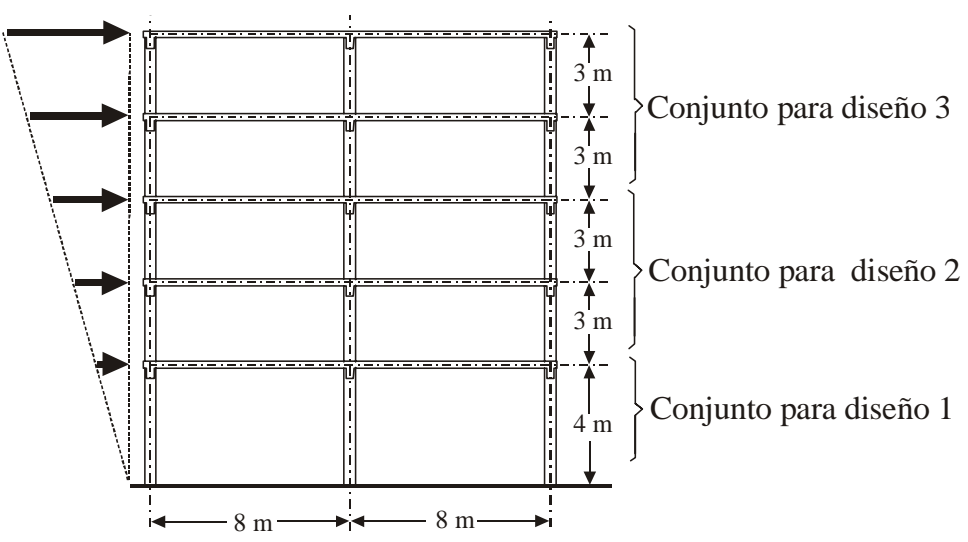

c) Elevación

Figura 1. Geometría del modelo

En lo que se refiere a los momentos de fluencia $M_{y}$ de trabes y columnas, se llevó a cabo un diseño por torsión para cada modelo, empleando las ecuaciones 1 y 2. Por tanto, estos momentos dependen de los valores seleccionados de $e, \alpha$ y $\delta$. En este trabajo se consideraron los siguientes valores: $e=0.0,0.05$ y $0.15 ; \alpha=1.0$ y 1.5 ; y $\delta=0.0,0.5$ y 1.0 . Dichos valores de excentricidad son típicos de estudios relacionados y los factores de amplificación son representativos de reglamentos de diseño conocidos (ICBO, 1997, NRCC, 1995). 
Respuesta torsional de edificios en el marco del análisis pushover

Tabla 1. Propiedades de las secciones transversales de los marcos de los modelos

\begin{tabular}{|c|c|c|c|c|}
\hline Planta & $\gamma$ & Entrepiso o nivel & Trabes [cm] & Columnas [cm] \\
\hline \multirow{3}{*}{ Cuadrada } & \multirow{3}{*}{0.5} & 1 & $30 \times 50$ & $50 \times 50$ \\
\hline & & 2 y 3 & $25 \times 50$ & $45 \times 45$ \\
\hline & & 4 y 5 & $25 \times 50$ & $35 \times 35$ \\
\hline \multirow{3}{*}{ Rectangular } & \multirow{3}{*}{0.8} & 1 & $30 \times 50$ & $50 \times 50$ \\
\hline & & 2 у 3 & $25 \times 50$ & $45 \times 45$ \\
\hline & & 4 y 5 & $25 \times 50$ & $35 \times 35$ \\
\hline
\end{tabular}

En relación con los diseños sísmicos de los modelos, se tomaron en cuenta las siguientes suposiciones para obtener los valores de $M_{y}$ de trabes y columnas para cada conjunto de diseño y para cada modelo (figura 1):

- El momento de fluencia $M_{y}$ se seleccionó como el máximo momento flexionante (para cada conjunto de diseño mostrado en la figura 1c) obtenido de un análisis elástico lineal de primer orden (redondeado al siguiente múltiplo de diez).

- En el caso general, los cortantes basales se aplicaron a lo largo de cada dirección ortogonal. La fuerza $X$ se aplicó de izquierda a derecha mientras que la fuerza paralela a $Y$ se consideró en ambas direcciones. Esto se debe a que la carga del análisis pushover también se aplicará en dirección positiva de $X$; sin embargo, para fines de diseño se desconoce el sentido de la fuerza ortogonal.

- Para fines de diseño, se aplicó un cortante en la base de $76.8 t$ con una distribución triangular con la altura (figura 1c). Esta magnitud se empleó para ambas plantas aun cuando el área de piso de la planta cuadrada es el doble de la de los modelos rectangulares. El uso de un solo cortante para ambas plantas permite que los resultados de ambas plantas sean comparables. El uso de un cortante para ambas plantas es posible ya sea variando el factor de reducción de carga lateral $(R)$ o la intensidad sísmica en el cálculo del cortante basal. La magnitud de esta fuerza resultó de suponer valores típicos de las cargas inerciales para el edificio y de la aceleración del terreno.

- No se incluyeron cargas verticales para la estimación de los momentos de diseño.

- Para la distribución de cargas laterales, cada losa se consideró con rigidez axial infinita.

- Similarmente, las columnas también se consideraron con rigidez axial infinita.

La mayoría de estas suposiciones han sido típicas de varios trabajos que estudian el problema de torsión en edificios (e.g., Kilar y Fajfar, 1997); por tanto, su incorporación en este estudio hace los resultados cualitativamente comparables a aquellos de trabajos relacionados con hipótesis similares. Es importante destacar que, a diferencia del programa descrito por Kilar y Fajfar (1997), la versión del programa que aquí se usa, toma en cuenta la interacción de momentos flexionantes ortogonales en columnas, como se describe en la siguiente sección.

Con referencia a los números de marcos de la figura 1, y tomando las fuerzas sísmicas paralelas al eje $X$ de izquierda a derecha, los momentos de fluencia de los marcos 1 y 3 resultaron como se resume en la tabla 2. En todos los casos, las fuerzas sísmicas paralelas a $Y$ siempre se sumaron porque para diseño su dirección es desconocida. Así, los marcos 1 y 3 tienen las mismas 
propiedades entre ellos y el centro de rotación se localiza a lo largo del marco 2, el cual no se afecta por torsión. Como se esperaba, los momentos de fluencia de los marcos 1 y 3 se incrementan con la excentricidad y con el coeficiente $\alpha$. En lo que se refiere al marco 4, al cual aplica la ecuación 2, los momentos de fluencia se reducen con $e$ y con $\delta$, como se muestra en la tabla 3. Para el marco 6, al cual aplica la ecuación 1, los momentos de fluencia se incrementan con $e$ y $\alpha$ (tabla 4).

Tabla 2. Momentos de fluencia $M_{y}$ de los elementos de los marcos 1 y $3(\delta=0.0)$

\begin{tabular}{|c|c|c|c|c|c|c|c|c|}
\hline Planta & $e$ & $\alpha$ & \multicolumn{3}{|c|}{$M_{y}$ en vigas [kg-m] } & \multicolumn{3}{c|}{$M_{y}$ en columnas [kg-m] } \\
\cline { 3 - 9 } & & & Grupo 1 & Grupo 2 & Grupo 3 & Grupo 1 & Grupo 2 & Grupo 3 \\
\hline \multirow{4}{*}{ Cuadrada } & 0.00 & - & 16,140 & 15,150 & 9,070 & 28,200 & 17,900 & 10,090 \\
\cline { 2 - 9 } & 0.05 & 1.0 & 17,350 & 16,290 & 9,750 & 30,320 & 19,240 & 10,900 \\
\cline { 2 - 9 } & & 1.5 & 17,960 & 16,860 & 10,090 & 31,430 & 19,920 & 11,310 \\
\cline { 2 - 9 } & 0.15 & 1.0 & 19,780 & 18,560 & 11,110 & 34,550 & 21,930 & 12,520 \\
\cline { 3 - 9 } & & 1.5 & 21,590 & 20,260 & 12,130 & 37,900 & 23,940 & 13,740 \\
\hline \multirow{5}{*}{ Rectangular } & 0.00 & - & 16,140 & 15,150 & 9,070 & 28,200 & 17,900 & 10,090 \\
\cline { 2 - 9 } & 0.05 & 1.0 & 16,860 & 15,760 & 9,470 & 29,560 & 18,590 & 11,320 \\
\cline { 3 - 9 } & 0.15 & 1.5 & 17,220 & 16,060 & 9,670 & 30,250 & 18,940 & 11,570 \\
\cline { 3 - 9 } & & 1.0 & 18,300 & 16,960 & 10,270 & 32,290 & 19,970 & 12,300 \\
\hline
\end{tabular}

Tabla 3. Momentos de fluencia $M_{y}$ de los elementos del marco $4(\alpha=1.0)$

\begin{tabular}{|c|c|c|c|c|c|c|c|c|}
\hline \multirow[t]{2}{*}{ Planta } & \multirow[t]{2}{*}{$e$} & \multirow[t]{2}{*}{$\delta$} & \multicolumn{3}{|c|}{$M_{y}$ en vigas [kg-m] } & \multicolumn{3}{|c|}{$M_{y}$ en columnas [kg-m] } \\
\hline & & & Grupo 1 & Grupo 2 & Grupo 3 & Grupo 1 & Grupo 2 & Grupo 3 \\
\hline \multirow{5}{*}{ Cuadrada } & 0.00 & - & 16,140 & 15,150 & 9,070 & 28,200 & 17,900 & 10,090 \\
\hline & \multirow[t]{2}{*}{0.05} & 0.5 & 15,540 & 14,580 & 8,730 & 27,120 & 17,230 & 9,680 \\
\hline & & 1.0 & 14,930 & 14,020 & 8,390 & 26,050 & 16,560 & 9,280 \\
\hline & \multirow[t]{2}{*}{0.15} & 0.5 & 14,330 & 13,450 & 8,050 & 24,970 & 15,890 & 8,870 \\
\hline & & 1.0 & 12,510 & 11,740 & 7,030 & 21,740 & 13,880 & 7,650 \\
\hline \multirow{5}{*}{ Rectangular } & 0.00 & - & 18,850 & 16,280 & 9,430 & 24,560 & 17,270 & 10,070 \\
\hline & \multirow[t]{2}{*}{0.05} & 0.5 & 17,650 & 15,220 & 8,830 & 23,030 & 16,140 & 9,430 \\
\hline & & 1.0 & 16,450 & 14,160 & 8,230 & 21,490 & 15,020 & 8,790 \\
\hline & \multirow[t]{2}{*}{0.15} & 0.5 & 15,250 & 13,100 & 7,620 & 19,950 & 13,890 & 8,150 \\
\hline & & 1.0 & 11,650 & 9,920 & 5,820 & 15,320 & 10,510 & 6,220 \\
\hline
\end{tabular}

Con esta información (resumida en la figura 1 y en las tablas 1 a 4), quedan completamente definidas las propiedades geométricas y de resistencia de los modelos para cada caso. En la siguiente sección, se analizan los modelos resultantes con un programa de análisis pushover clásico tridimensional. 
Respuesta torsional de edificios en el marco del análisis pushover

Tabla 4. Momentos de fluencia $M_{y}$ de los elementos del marco 6 (calculados con $\delta=0.0$ )

\begin{tabular}{|c|c|c|c|c|c|c|c|c|}
\hline \multirow[t]{2}{*}{ Planta } & \multirow[t]{2}{*}{$e$} & \multirow[t]{2}{*}{$\alpha$} & \multicolumn{3}{|c|}{$M_{y}$ en vigas [kg-m] } & \multicolumn{3}{|c|}{$M_{y}$ en columnas [kg-m] } \\
\hline & & & Grupo 1 & Grupo 2 & Grupo 3 & Grupo 1 & Grupo 2 & Grupo 3 \\
\hline \multirow{5}{*}{ Cuadrada } & 0.00 & - & 16,140 & 15,150 & 9,070 & 28,200 & 17,900 & 10,090 \\
\hline & \multirow{2}{*}{0.05} & 1.0 & 17,350 & 16,290 & 9,750 & 30,320 & 19,240 & 11,650 \\
\hline & & 1.5 & 17,960 & 16,860 & 10,090 & 31,430 & 19,920 & 11,310 \\
\hline & \multirow[t]{2}{*}{0.15} & 1.0 & 19,770 & 18,560 & 11,110 & 34,550 & 21,930 & 13,270 \\
\hline & & 1.5 & 21,590 & 20,260 & 12,130 & 37,900 & 23,940 & 13,740 \\
\hline \multirow{5}{*}{ Rectangular } & 0.00 & - & 18,850 & 16,280 & 9,430 & 24,560 & 17,270 & 10,070 \\
\hline & \multirow[t]{2}{*}{0.05} & 1.0 & 21,260 & 18,400 & 10,640 & 27,650 & 19,520 & 11,350 \\
\hline & & 1.5 & 22,460 & 19,470 & 11,240 & 29,190 & 20,650 & 11,990 \\
\hline & \multirow[t]{2}{*}{0.15} & 1.0 & 26,060 & 22,650 & 13,050 & 33,820 & 24,030 & 13,910 \\
\hline & & 1.5 & 29,660 & 25,830 & 14,850 & 38,440 & 27,400 & 15,830 \\
\hline
\end{tabular}

\section{RESULTADOS DEL ANÁLISIS PUSHOVER}

Para llevar a cabo el análisis pushover, se adaptó un programa desarrollado previamente (De la Colina, 2004) para tomar en cuenta la interacción biaxial no lineal en las columnas. El programa no considera el efecto de las variaciones de carga axial; sin embargo, para edificios de baja altura donde el análisis pushover clásico pudiera aplicar, las variaciones de carga axial en columnas son pequeñas. El programa considera la interacción de momentos flexionantes en columnas reduciendo los momentos de fluencia independientes alrededor de cada uno de los ejes principales de la sección de la columna que se considere con ayuda de una curva de fluencia elíptica, como se describe en los párrafos siguientes. En todos los casos de este trabajo, se consideró un comportamiento bilineal con la rigidez post fluencia igual a 5\% de la rigidez inicial.

La interacción biaxial de momentos en las columnas se considera como se describe a continuación, con ayuda de la figura 2. En ésta, la elipse en el plano $\mathbf{M}_{1}-\mathbf{M}_{2}$ representa la combinación de momentos alrededor de cada uno de los ejes principales de las columnas que ocasiona fluencia en una sección transversal. Así el punto A, por ejemplo, indica el valor del momento de fluencia para el caso de flexión uniaxial alrededor de un eje principal de la sección de la columna. Similarmente, el punto B representa el momento de fluencia uniaxial alrededor del eje ortogonal. Los momentos $\mathrm{M}_{1}$ correspondiente al punto $\mathrm{B}$ y el $\mathrm{M}_{2}$ correspondiente al punto A se pueden calcular fácilmente para una sección dada (y un nivel de carga axial fijo). Aquí, estos momentos se proponen con los valores de $\mathrm{M}_{\mathrm{y}}$ calculados en la sección anterior. Los puntos A y B definen la ecuación de la elipse en el plano de momentos.

A la derecha de la elipse en la misma figura, se muestra la relación momento-rotación alrededor de un eje principal de la sección transversal. En éste, el punto B' define el momento de fluencia correspondiente a la condición de flexión uniaxial (sin interacción). Similarmente, en la parte inferior de la elipse se presenta el diagrama momento-rotación para el eje ortogonal de la sección.

En el análisis pushover clásico, se supone que tanto el patrón de cargas como la relación de los cortantes en ambas direcciones ortogonales del edificio se mantienen invariantes. Por tanto, al aplicar las primeras cargas laterales, las cuales generalmente no causan fluencia en la 
estructura, es posible estimar los momentos que se inducen en la sección alrededor de cada eje principal de la sección de la columna.

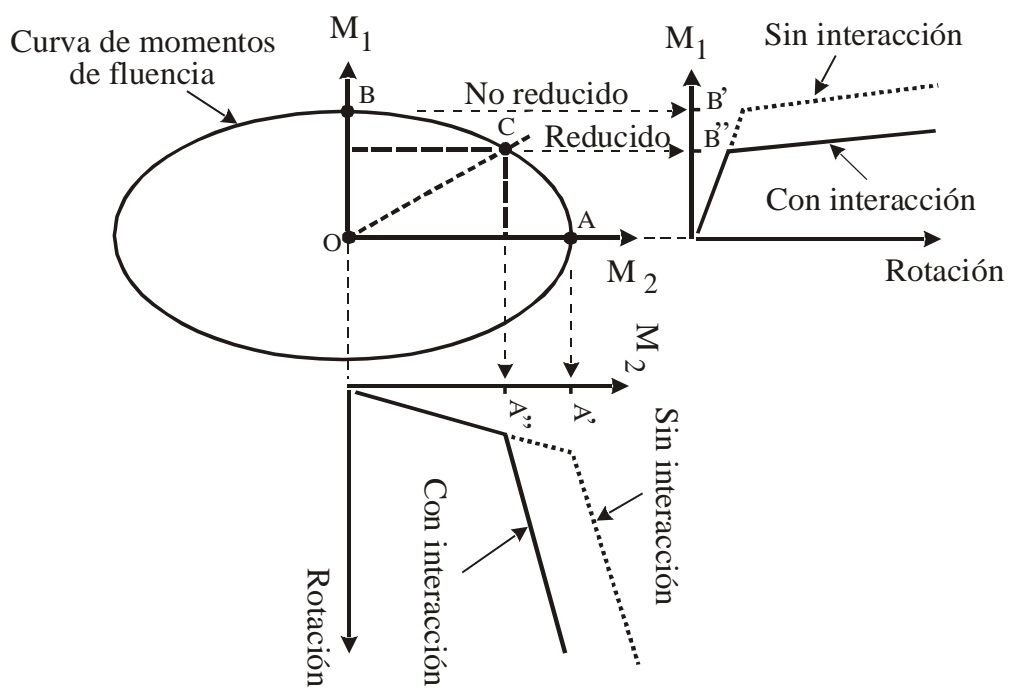

Figura 2. Interacción de momentos flexionantes en columnas

Debido a que los subsecuentes cortantes laterales que no causan fluencia mantienen una relación invariante, es posible conocer la pendiente de la línea OC en el plano de momentos. La intersección de la recta OC con la elipse define el punto donde eventualmente se tendrá la fluencia correspondiente al caso biaxial (punto C). Con este punto, los diagramas momentorotación para el caso de flexión biaxial (con interacción) quedan definidos. Estos últimos se emplean para la revisión de fluencia alrededor de cada eje de la sección, manteniendo la misma rigidez post fluencia. Como se observa, la interacción biaxial de momentos, se reduce al empleo de los diagramas momento-rotación indicados con línea continua (con interacción) en lugar de usar los correspondientes al caso uniaxial. Para el modelado inelástico de trabes y columnas, se emplea el modelo de una componente de Giberson (1969), el cual supone que la inelasticidad se concentra en los extremos de los elementos. Este modelo y el procedimiento de análisis basado en el método de rigidez se implementaron en un programa de cálculo en lenguaje de programación C. Básicamente, este programa incorpora la interacción biaxial de columnas al trabajo previo presentado por De la Colina (2004).

En la parte inicial de esta sección, se presenta como marco de referencia la respuesta global de los sistemas torsionalmente desbalanceados $(e>0.0)$. Posteriormente, se expone el efecto de los factores relacionados con el diseño por torsión $\alpha$ y $\delta$. Finalmente, se muestra el efecto al aplicar simultáneamente dos cortantes basales ortogonales para tres valores del cociente $\Omega(0.0,0.4$ y 1.0$)$, donde $\Omega=$ cociente del cortante basal a lo largo de la dirección $Y$ entre el cortante basal a lo largo de la dirección $X$. La respuesta estática se da en términos de los siguientes parámetros de respuesta: 1) fuerza cortante vs desplazamiento lateral del centro de masa de la losa superior del edificio, 2) desplazamientos laterales de los marcos, 3) mecanismos plásticos y 4) energía plástica disipada por cada uno de los marcos. 


\section{Respuesta de sistemas torsionalmente desbalanceados - efecto de la excentricidad}

Con la idea de tener un marco de referencia, se presenta primero la respuesta de los modelos torsionalmente balanceados (TB) $(e=0.0)$ junto con la respuesta de sistemas TD $(e=0.05 \mathrm{y}$ 0.15 ) diseñados con $\alpha=1.0$ y $\delta=0.0$. En la figura 3 se muestran las curvas pushover, las cuales relacionan el cortante basal aplicado vs. el desplazamiento lateral del centro de masa de la losa superior de edificio. Tradicionalmente, estos son los principales resultados que se obtienen del análisis pushover. Dichas curvas se calcularon hasta un cortante basal de 100 t. Como se muestra adelante, este cortante basal conduce a valores de la ductilidad de desplazamiento de entre dos y tres, los cuales son consistentes con valores típicos del factor de reducción de fuerzas laterales $(R)$. Los modelos correspondientes a las curvas de la figura 3 no alcanzaron a desarrollar un mecanismo plástico a esta magnitud de carga. Se observa que los sistemas TD (pero diseñados contra torsión) resultaron con una capacidad de cortante basal mayor que en el caso del sistema TB. El desplazamiento lateral relativo a la altura del edificio (drift) resultó aproximadamente del 1\%. Este desplazamiento relativo, sin embargo, no se considera aquí como un valor crítico porque el programa no toma en cuenta las características particulares en los extremos de los miembros (e.g., detalles de refuerzo). Así, el propósito de estas curvas pushover es sólo mostrar que dichas curvas de los sistemas TD se ubican por arriba de la curva pushover del correspondiente sistema TB con $e=0.0$. Por ello, la magnitud del desplazamiento lateral se toma aquí sólo como un resultado secundario. Como se esperaba, las figuras corroboran que los sistemas se comportaron lineales hasta el cortante basal de diseño $\left(V_{b}=76.8 t\right)$.

Las curvas pushover anteriores del centro de masa de la losa de azotea son útiles en el sentido de que muestran la respuesta global del sistema. Ellas no muestran la respuesta particular de sus subestructuras (marcos en este caso). Sin embargo, se pueden construir fácilmente gráficas de los desplazamientos de entrepiso de cada marco tomando ventaja del movimiento de cuerpo rígido de las losas.

Los desplazamientos relativos laterales de los marcos se muestran en la figura 4 para los marcos paralelos al eje $X$ de los modelos diseñados con $\alpha=1.0$ y $\delta=0.0$. En esta figura, se identifican los desplazamientos de los sistemas TB, los cuales pueden tomarse como desplazamientos de referencia, con círculos blancos unidos con líneas intermitentes. Como se esperaba para ambas plantas (cuadradas y rectangulares), estos desplazamientos de sistemas TB $(e=0.0)$ son iguales para los tres marcos de la misma planta. Los resultados de ambas plantas muestran que los desplazamientos de los marcos de los edificios TD (pero diseñados contra torsión con $\alpha=1.0$ y $\delta=0.0$ ) son más pequeños que aquellos correspondientes al sistema TB. Este resultado se debe al hecho de que los edificios diseñados por torsión son más resistentes que los sistemas TB y, por tanto, entre mayor sea la excentricidad menor serán los desplazamientos, a pesar de que la torsión esté presente. Estos resultados corresponden a un análisis pushover (estático) pero son consistentes con los resultados obtenidos de análisis no lineales dinámicos de sistemas TD de un piso (e.g., De-la-Colina, 1999), en el sentido de que, para una selección dada de factores de torsión, se obtiene una respuesta menor para sistemas con excentricidad grande. 
Jaime De la Colina Martínez


Figura 3. Curvas pushover de modelos diseñados con $\alpha=1.0$ y $\delta=0.0$.

Otro resultado que también se puede obtener de un análisis pushover es la distribución de comportamiento no lineal en los marcos. Por ejemplo, la figura 5 muestra estas distribuciones para los marcos paralelos al eje correspondientes a un cortante basal de $100 \mathrm{t}$. En estos esquemas, los círculos representan los extremos de los miembros donde se presentan no linealidades. Más aún, el diámetro de cada círculo es proporcional a la ductilidad de rotación calculada para ese extremo. Asimismo, se puede observar en esta figura que las distribuciones de no linealidades son similares entre los marcos orientados en la misma dirección. Sin embargo, debe resultar claro que esta observación pudiera no ser válida si los marcos no son también similares en su geometría o en su distribución de resistencias. A primera vista, no es claro cuál marco ha incursionado más en el comportamiento no lineal. Esto sugiere la necesidad de incorporar un parámetro de respuesta complementario. 

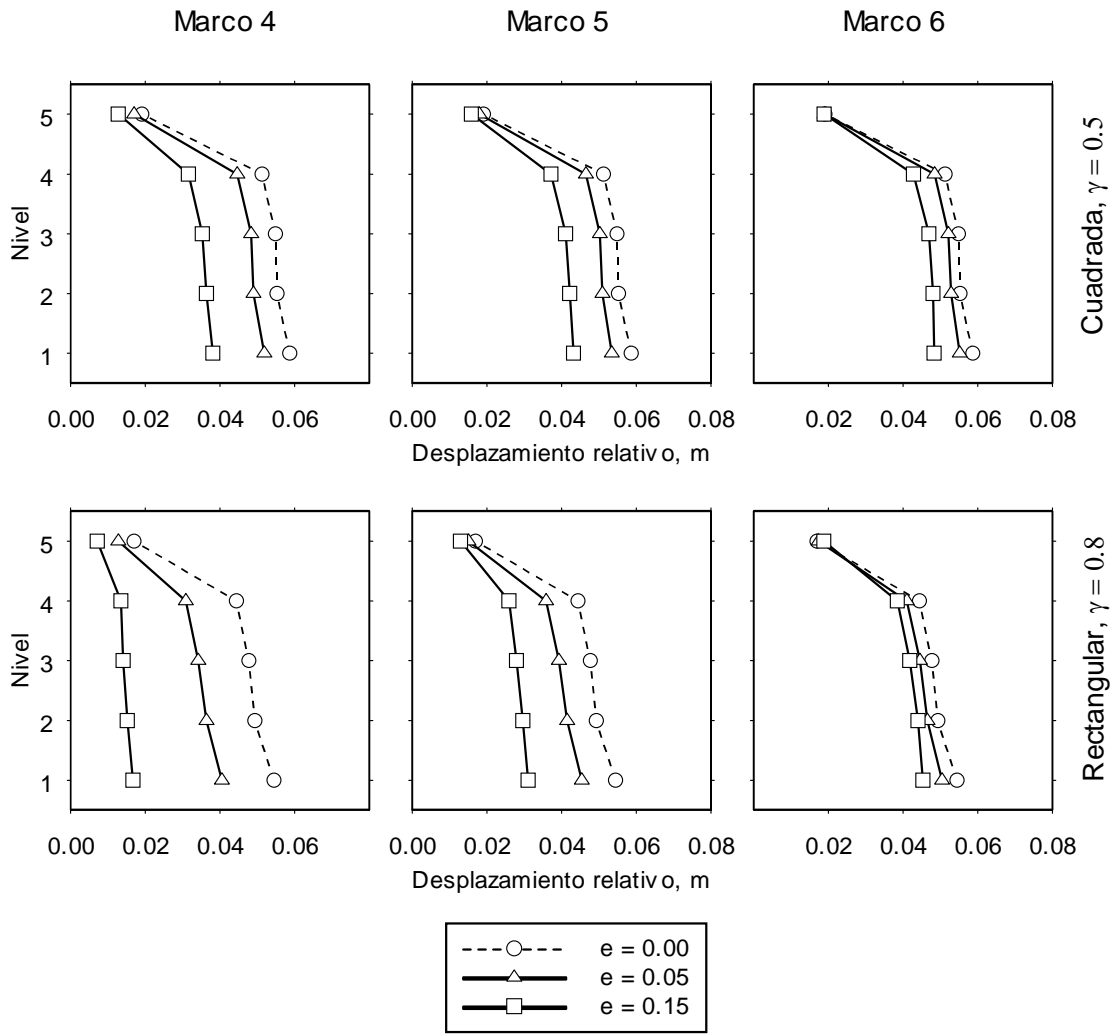

Figura 4. Desplazamientos relativos de modelos diseñados con $\alpha=1.0$ y $\delta=0.0$

Para ayudar a resolver este problema de identificar el marco con el mayor comportamiento no lineal, se incorpora la energía plástica disipada $E_{p}$, la cual se calcula aquí como el área bajo la curva que relaciona el momento flexionante con la rotación plástica de un extremo de un elemento dado (ver figura 6). Cuando el valor de este parámetro se suma para todos los extremos de los elementos de un marco, se obtiene la energía plástica disipada por el marco, la cual puede usarse para valorar el comportamiento no lineal del marco. La tabla 5 contiene las energías plásticas disipadas para los marcos paralelos al eje $X$. Primeramente se puede observar, que la energía plástica disminuye para valores crecientes de la excentricidad. Si no se considerara la torsión en el diseño, las energías plásticas pudieran incrementarse para aquellos marcos no favorecidos por la torsión (ver tabla 6, la cual lista estas energías para sistemas sin diseño por torsión). Nótese en la tabla 6 que para el marco 5, el cual contiene al centro de rotación, las energías plásticas no cambian significativamente. La reducción de la energía disipada del marco 4 observada en la tabla 6, para sistemas con excentricidad creciente, se debe al efecto favorable de la torsión en este marco. 


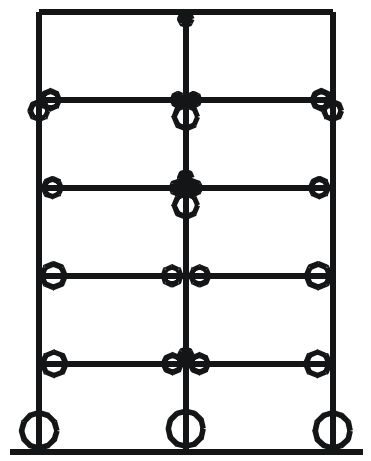

Marco 4

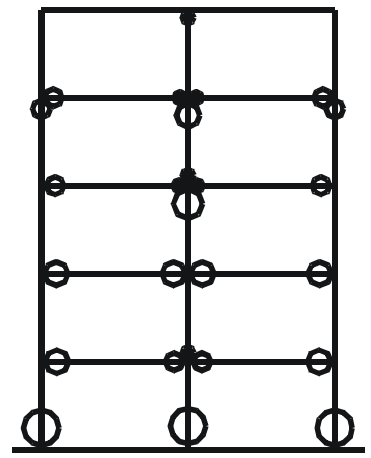

Marco 5

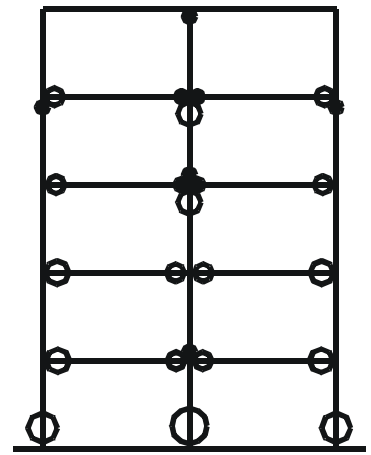

Marco 6

a) Planta cuadrada $\gamma=0.5, V_{\max }=100 \mathrm{t}$

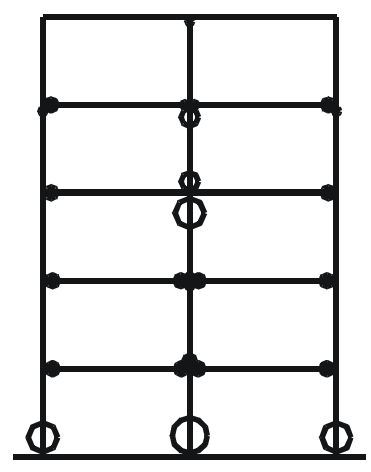

Marco 4

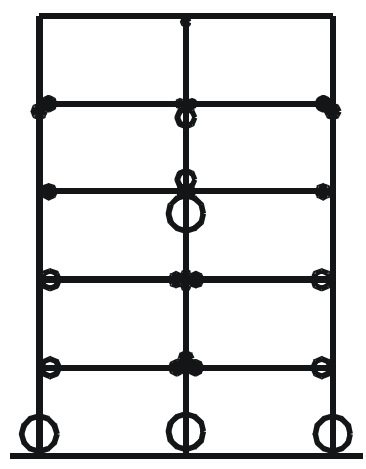

Marco 5

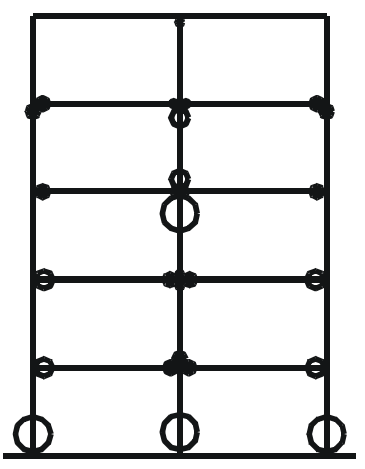

Marco 6

b) Planta ectangular $\gamma=0.8, V_{\max }=100 \mathrm{t}$

Figura 5. Distribución de no linealidades en modelos diseñados con $e=0.05$, diseñados con $\quad \alpha=1.0$ y $\delta=0.0$

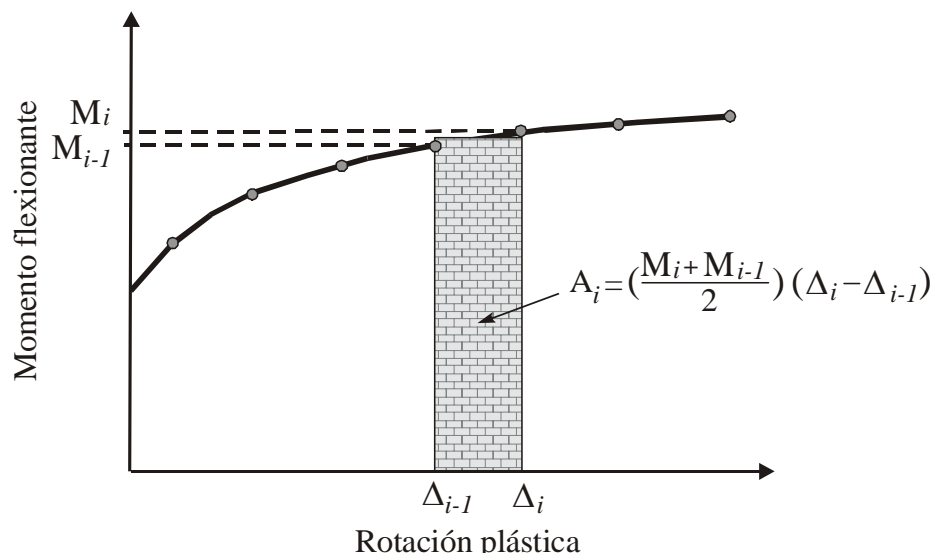

Figura 6. Energía plástica $E_{p}$ en un extremo de un elemento 
Respuesta torsional de edificios en el marco del análisis pushover

Tabla 5. $E_{p}$ disipada para $V_{b}=100 \mathrm{t}$ en sistemas diseñados con $\alpha=1.0$ y $\delta=0.0\left(E_{p}\right.$ en kg-m)

\begin{tabular}{|c|c|c|c|c|}
\hline Planta y valor $\delta$ & $\begin{array}{c}\text { excentricidad } \\
e\end{array}$ & Marco 4 & Marco 5 & Marco 6 \\
\hline \multirow{3}{*}{ Cuadrada, 0.5 } & 0.00 & 3,927 & 3,927 & 3,927 \\
\cline { 2 - 5 } & 0.05 & 3,260 & 3,445 & 3,772 \\
\cline { 2 - 5 } & 0.15 & 1,976 & 2,510 & 3,328 \\
\hline \multirow{3}{*}{ Rectangular, 0.8 } & 0.00 & 3,351 & 3,351 & 3,351 \\
\cline { 2 - 5 } & 0.05 & 2,002 & 2,493 & 3,146 \\
\cline { 2 - 5 } & 0.15 & 146 & 1,316 & 2,880 \\
\hline
\end{tabular}

Tabla 6. $E_{p}$ disipada para $V_{b}=100 \mathrm{t}$ en sistemas sin diseño por torsión ( $E_{p}$ in kg-m).

\begin{tabular}{|c|c|c|c|c|}
\hline Planta y valor $\delta$ & $\begin{array}{c}\text { excentricidad } \\
e\end{array}$ & Marco 4 & Marco 5 & Marco 6 \\
\hline \multirow{3}{*}{ Cuadrada, 0.5 } & 0.00 & 3,927 & 3,927 & 3,927 \\
\cline { 2 - 5 } & 0.05 & 3,693 & 3,923 & 4,131 \\
\cline { 2 - 5 } & 0.15 & 3,241 & 3,903 & 4,499 \\
\hline \multirow{3}{*}{ Rectangular, 0.8 } & 0.00 & 3,351 & 3,351 & 3,351 \\
\cline { 2 - 5 } & 0.05 & 2,585 & 3,351 & 4,111 \\
\cline { 2 - 5 } & 0.15 & 1,215 & 3,347 & 5,549 \\
\hline
\end{tabular}

Los resultados mostrados en este apartado ilustran el tipo de respuestas (salidas) que se pueden obtener de un análisis pushover. Así, no sólo las curvas pushover ofrecen información del comportamiento de la estructura. Los desplazamientos de los marcos, las distribuciones de comportamiento no lineal y las energías plásticas disipadas por los marcos también proveen información que puede ser útil para el diseño y/o la evaluación de estructuras. Como se indicó antes, los resultados pushover no son definitivos y requieren de una validación complementaria, particularmente para edificios donde la contribución de modos superiores de vibración puede ser importante.

\section{Efectos de los factores de diseño por torsión $\alpha$ y $\delta$}

En el apartado anterior, se mostraron los resultados de análisis pushover para sistemas TD ( $e>$ 0.0 ) diseñados con $\alpha=1.0$ y $\delta=0.0$. Aquí se presentan los resultados calculados en sistemas diseñados con otros valores de estos parámetros de diseño. El principal objetivo de este apartado es observar cuáles de las conclusiones obtenidas previamente de estudios similares (con respecto al efecto de estos factores) empleando análisis dinámicos no lineales, se pueden obtener a partir de análisis pushover. Primeramente, se muestra el efecto del factor $\alpha$ usando sistemas diseñados con un valor constante de $\delta=0.0$. Posteriormente, se muestra el efecto de $\delta$ usando un valor constante de $\alpha=1.0$.

Para ilustrar el efecto de $\alpha$, las figuras 7 y 8 (para sistemas con $e=0.05$ y 0.15 , respectivamente) muestran los desplazamientos relativos laterales de los marcos de sistemas 
diseñados con dos valores de este factor. En todos los casos, el cortante basal máximo aplicado sobre los modelos fue de $100 \mathrm{t}$. Las gráficas en estas figuras ilustran que ambos valores $\alpha=1.0 \mathrm{o}$ $\alpha=1.5$ son capaces de mantener los desplazamientos (estáticos) laterales debajo de los valores de desplazamiento de los sistemas TB $(e=0.0)$. Como se esperaba, los desplazamientos críticos corresponden a aquellos del marco 6 donde las cortantes por torsión se suman a aquellos debidos a cortante. Los desplazamientos del marco 4 quedan gobernados por el factor $\delta$, como se muestra abajo. Contrastando los resultados de la figura 7 para $e=0.05$ con aquellos de la figura 8 para $e=$ 0.15 , se puede observar que el factor $\alpha$ conduce a mayores márgenes de seguridad en sistemas con excentricidad grande que en sistemas con excentricidad pequeña. Estos resultados coinciden con los obtenidos de análisis dinámicos no lineales de modelos de edificios de baja altura (De-laColina, 2003).

Marco 4
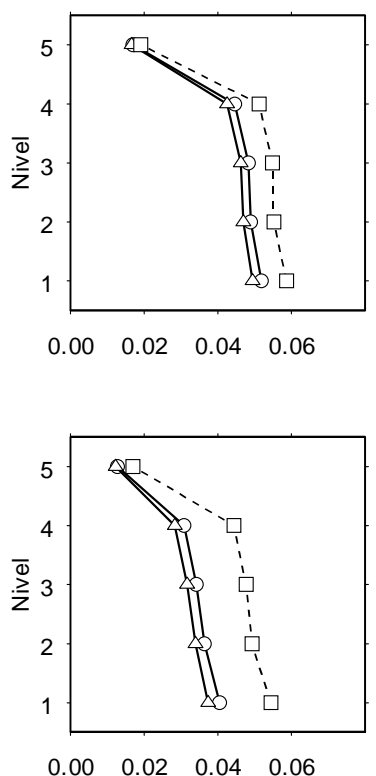

Marco 5
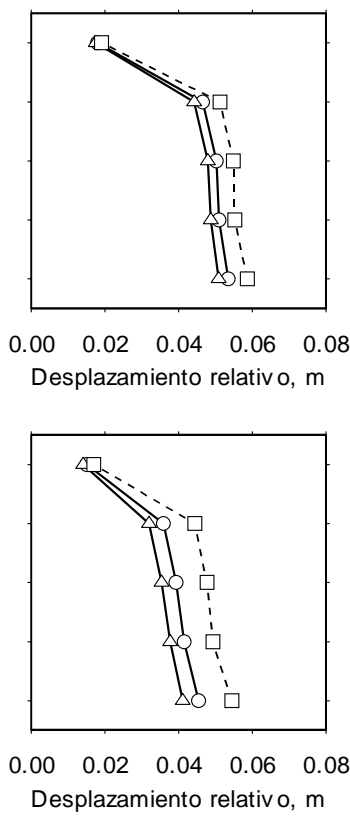

$$
\begin{array}{ll}
\longrightarrow \longrightarrow & \begin{array}{l}
\alpha=1.0 \\
\alpha=1.5
\end{array} \\
-\square-- & \begin{array}{l}
\text { Referencia, e }=0.0
\end{array}
\end{array}
$$

Marco 6
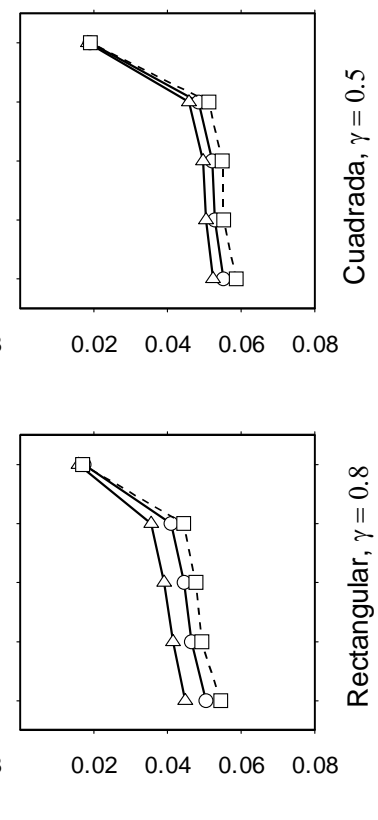

Figura 7. Desplazamientos relativos de modelos con $e=0.05$ diseñados con $\delta=0.0$

El efecto del factor $\delta$ en la ecuación 2 puede observarse en la figura 9, donde los desplazamientos laterales del marco se calcularon para los sistemas TD $(e=0.15)$ diseñados con tres valores de este parámetro y con $\alpha=1.0$. Como se observó previamente en estudios dinámicos realizados por Wong y Tso (1995) con modelos de un piso y por De-la-Colina (2003) con modelos de cinco niveles, las curvas de la figura 9 coinciden con la idea de que el valor de $\delta$ $=1.0$ no es conservador para diseño. Para ambas plantas, los desplazamientos del marco 6 resultaron más grandes que aquellos calculados para el sistema TB. Consecuentemente, puede observarse que los sistemas diseñados con $\delta=0.5$ mantienen los desplazamientos por debajo de 
aquellos del sistema TB. Este último resultado también es consistente con aquellos obtenidos en las dos referencias anteriores. Todos estos resultados sugieren que el uso del factor $\delta=0.0$ parece ser demasiado conservador.

\section{Efectos del cortante basal ortogonal}

En este apartado se estudia el efecto de aplicar de manera simultánea dos cortantes basales ortogonales. Además del cortante basal en la dirección $X\left(V_{x}\right)$, se aplica un cortante ortogonal $V_{y}$ $=\Omega \cdot V_{x}$ con dos valores no nulos de $\Omega(0.4 \mathrm{y} 1.0)$. Antes de presentar los resultados, es importante tener en mente que los marcos en dirección $Y$ fueron diseñados sumando el cortante basal a lo largo de esta dirección $Y$ y los cortantes causados por torsión cuando la fuerza paralela a $X$ actúa con excentricidad. Asimismo, es importante recordar que el programa de análisis que se usó para este trabajo, toma en cuenta la interacción no lineal de los momentos flexionantes ortogonales en columnas. Así, los cortantes basales $\left(V_{x} \mathrm{y} V_{y}\right)$ interactúan en dos formas: primero, por la adición de fuerzas causadas por cortante y por torsión y 2) por la interacción no lineal de momentos flexionantes en cada columna.
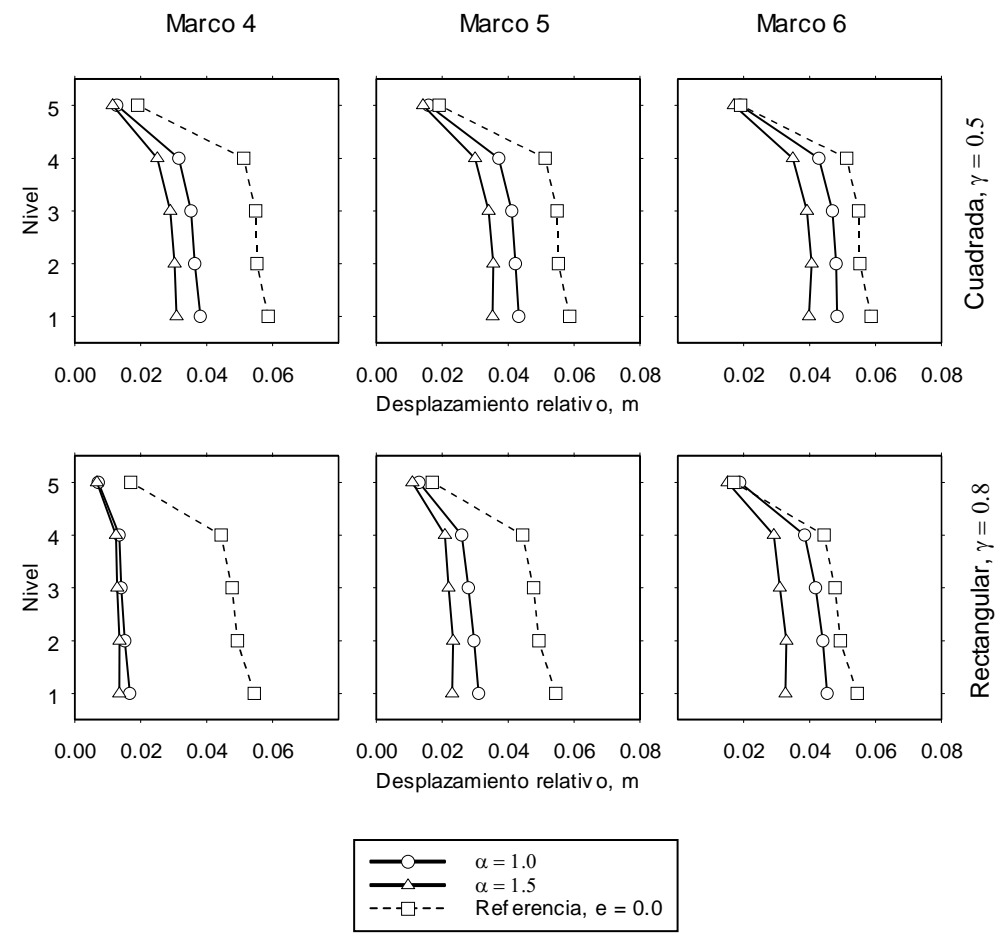

Figura 8. Desplazamientos relativos de modelos con $e=0.15$ diseñados con $\delta=0.0$. 

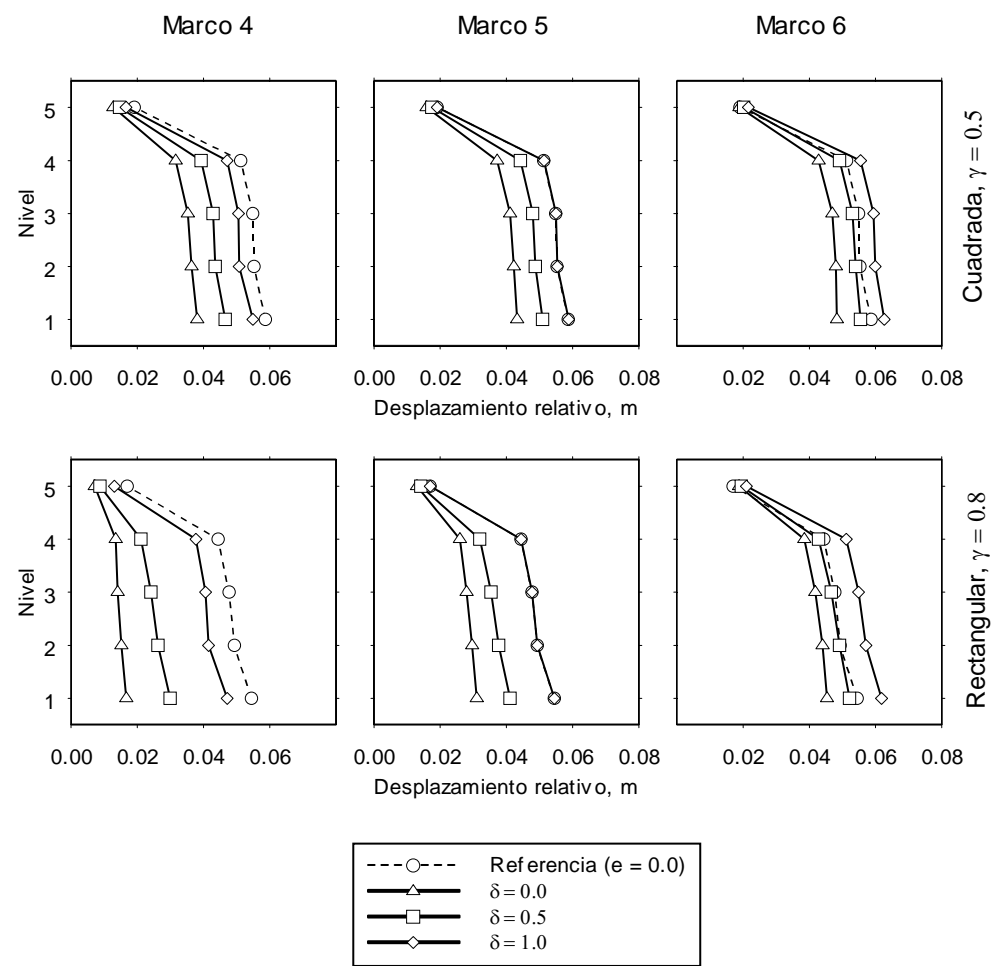

Figura 9. Desplazamientos relativos de modelos con $e=0.15$ diseñados con $\alpha=1.0$.

Las figuras 10 y 11 muestran las curvas pushover para modelos con $e=0.05$ y $e=0.15$, respectivamente, diseñados con $\alpha=1.0$ y $\delta=0.0$. Dichas figuras muestran claramente que, para los casos considerados, las respuestas para $\Omega=0.0$ y $\Omega=0.4$ son prácticamente las mismas. En otras palabras, los resultados indican que el cortante ortogonal no parece ser importante si su magnitud es igual o menor del $40 \%$ del cortante basal que causa torsión en el sistema. Los resultados sugieren que esta observación es independiente de: 1) el valor de la excentricidad, 2) de la relación de aspecto de la planta y 3) del valor del cociente de rigidez torsional $\gamma$. Sin embargo, resultados de análisis dinámicos (Valdés, 2004) indican que la componente ortogonal tiene un efecto mayor que el observado en este trabajo.

Así, el cortante ortogonal se vuelve importante cuando su magnitud es cercana a la del cortante que causa torsión. Las mismas conclusiones se observan en las figuras 12 y 13, las cuales muestran los desplazamientos laterales de los marcos de los modelos con $e=0.05$ y $e=$ 0.15 diseñados con $\alpha=1.0$ y $\delta=0.0$. En estas gráficas, los desplazamientos laterales calculados con $\Omega=1.0$ son significativamente diferentes de aquellos obtenidos con $\Omega=0.0$ o con $\Omega=0.4$. 
Respuesta torsional de edificios en el marco del análisis pushover
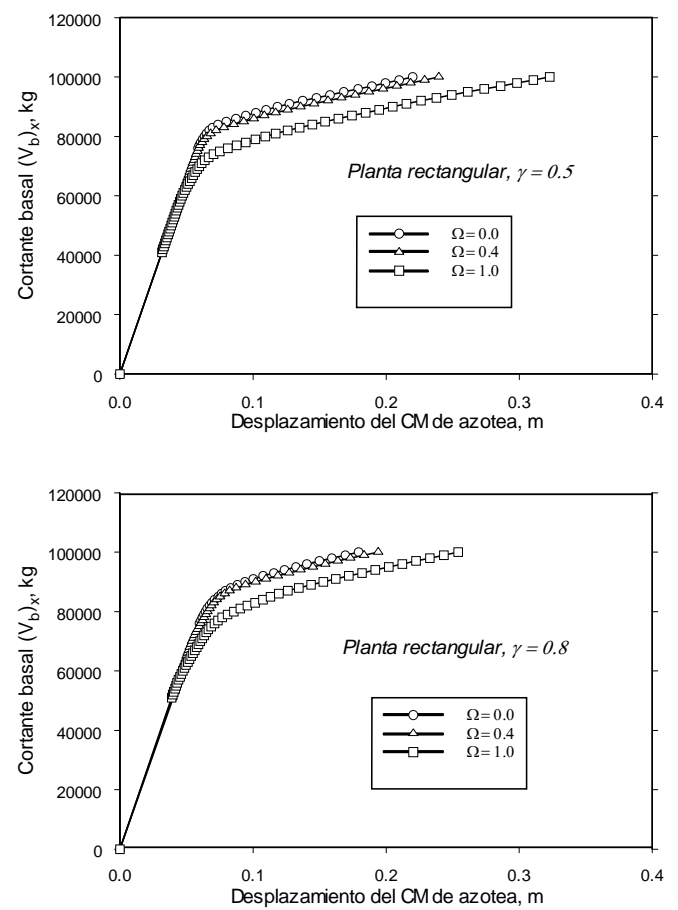

Figura 10. Curvas pushover para modelos con $e=0.05$ diseñados con $\alpha=1.0$ y $\delta=0.0$
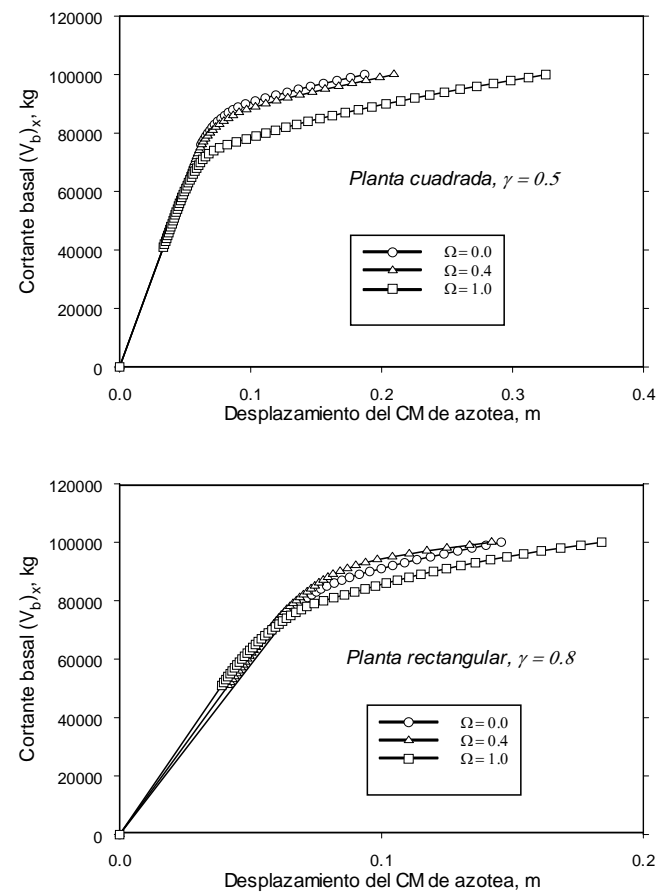

Figura 11. Curvas pushover para modelos con $e=0.15$ diseñados con $\alpha=1.0$ y $\delta=0.0$ 

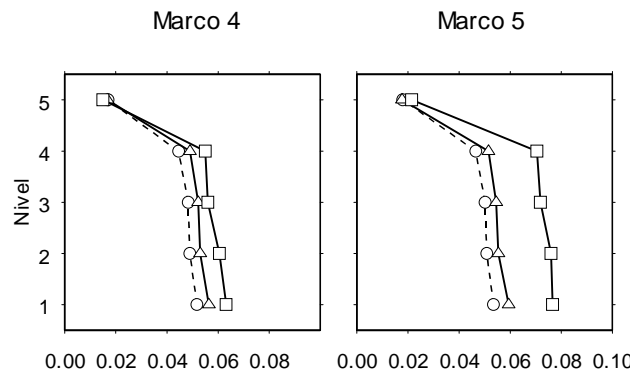

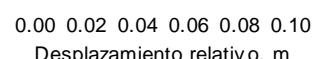

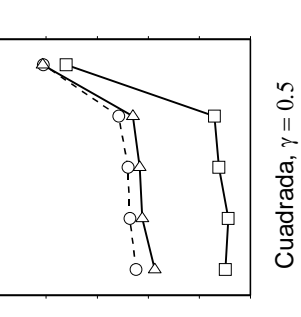

Desplazamiento relativ $\mathrm{o}, \mathrm{m}$

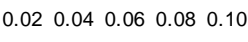
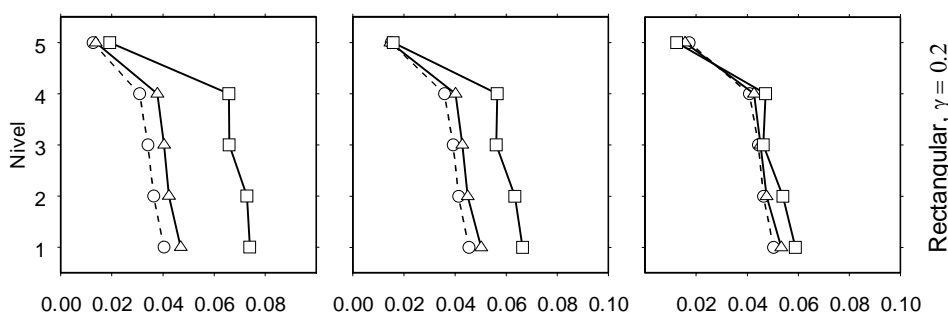

$0.00 \quad 0.02 \quad 0.040 .06 \quad 0.08$

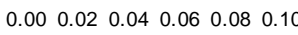
Desplazamiento relativ o, $\mathrm{m}$

$$
\begin{array}{|cc}
--O-- & \Omega=0.0 \\
\longrightarrow & \Omega=0.4 \\
\square & \Omega=1.0
\end{array}
$$

Figura 12. Desplazamientos laterales de modelos con $e=0.05$ diseñados con $\alpha=1.0$ y $\delta=0.0$
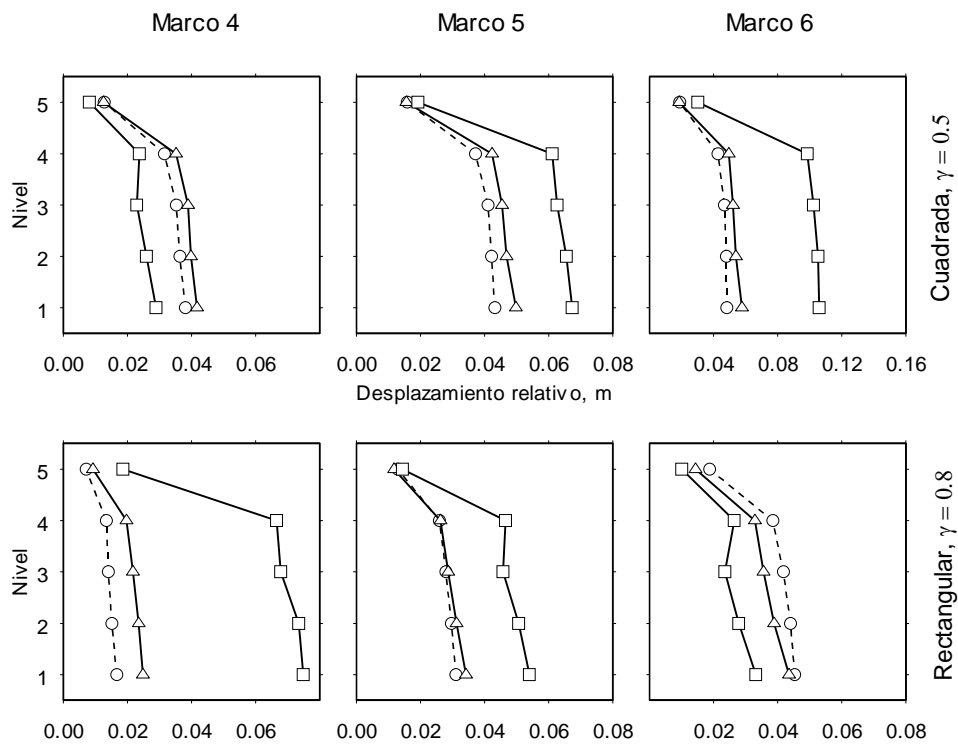

Desplazamiento relativ o, $\mathrm{m}$
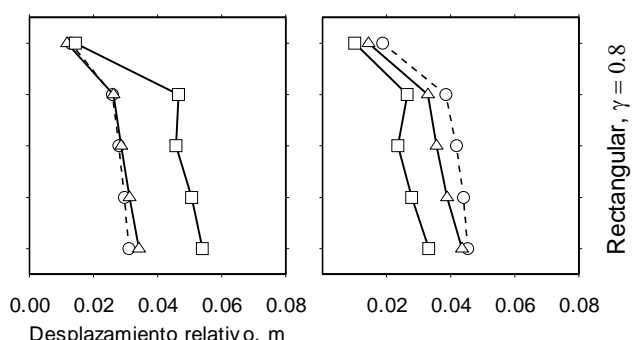

$$
\begin{array}{ll}
--\mathrm{O--} & \Omega=0.0 \\
\longrightarrow & \Omega=0.4 \\
\square- & \Omega=1.0
\end{array}
$$

Figura 13. Desplazamientos laterales de modelos con $e=0.15$ diseñados con $\alpha=1.0$ y $\delta=0.0$ 


\section{CONCLUSIONES}

Se llevaron a cabo análisis tipo pushover (estáticos, no lineales incrementales) en modelos tridimensionales a base de marcos de cinco niveles. El trabajo se enfocó en observar cómo interpreta el análisis pushover clásico algunas de las variables relacionadas con el problema de torsión en edificios y para identificar si los resultados con este tipo de análisis son consistentes con los obtenidos de estudios dinámicos en sistemas torsionalmente desbalanceados (TD) efectuados previamente por otros investigadores. Los resultados aquí presentados no pretenden validar las respuestas calculadas con la metodología del análisis pushover con aquellas obtenidas de análisis dinámicos no lineales. El estudio se orienta a ofrecer resultados para apoyar la hipótesis de que el análisis pushover puede ser una herramienta efectiva para el diseño o evaluación de edificios TD de baja altura. Las principales conclusiones derivadas de estos resultados se presentan enseguida.

Los resultados indican que el factor para diseño por torsión $\alpha$ (ec 1) provee mayor margen de seguridad a sistemas con excentricidad grande que a sistemas con excentricidad pequeña. Este resultado es consistente con las observaciones derivadas de estudios dinámicos no lineales.

Los resultados del análisis pushover indican que los sistemas diseñados con $\delta=0.5$ mantienen las magnitudes de los desplazamientos laterales de los marcos cercanas (o menores) que aquellas de los correspondientes sistemas torsionamente balanceados (TB). Estos resultados también coinciden con aquellos obtenidos de estudios dinámicos no lineales.

La energía plástica disipada, la cual ha mostrado ser un parámetro complementario obtenido del análisis pushover, disminuye con los valores de la excentricidad en sistemas diseñados por torsión. Para los casos considerados, donde la geometría y la distribución de resistencias son similares entre marcos paralelos, los esquemas de distribución de comportamiento no lineal entre estos marcos también resultaron similares.

Las curvas pushover y los desplazamientos laterales de los marcos de respuestas para $\Omega=$ 0.0 y $\Omega=0.4$ son prácticamente las mismas. A pesar de que se consideró en el análisis pushover la interacción no lineal de momentos flexionantes, los resultados muestran que si el cortante ortogonal es $40 \%$ o menor que el cortante de las fuerzas que causan torsión, la aplicación simultánea de estos cortantes puede despreciarse en el cálculo de la respuesta.

En general, los resultados obtenidos del análisis pushover son consistentes con aquellos obtenidos de análisis dinámicos no lineales. Esta conclusión apoya la hipótesis que el análisis pushover puede ser útil para el diseño y la evaluación de edificios a base de marcos torsionamente desbalanceados de baja altura.

En este trabajo se consideraron los siguientes valores: $e=0.0,0.05$ y $0.15 ; \alpha=1.0$ y 1.5 ; y $\delta=0.0,0.5$ y 1.0. Dichos valores de excentricidad son típicos de estudios relacionados y los factores de amplificación son representativos de reglamentos de diseño conocidos (ICBO, 1997, NRCC, 1995). 


\section{AGRADECIMIENTOS}

El autor agradece el apoyo de la Secretaría de Investigación y Estudios Avanzados de la Universidad Autónoma del Estado de México.

\section{REFERENCIAS}

Alcocer, S, (1986). "Centro de torsión de edificios obtenido matricialmente". Tesis de licenciatura, Universidad Nacional Autónoma de México.

Applied Technology Council (ATC), (2004). "Improvement of nonlinear static seismic analysis procedures (FEMA-440)”, Federal Emergency Management Agency, Washington, DC

Bracci, J M, S K Kunnath y A M Reinhorn, (1997). "Seismic performance and retrofit evaluation for reinforced concrete structures”, Journal of Structural Engineering, ASCE, 123, No. 1, 3-10.

Building Seismic Safety Council (BSSC), (1997). "NEHRP Guidelines for the Seismic Rehabilitation of Buildings, FEMA-273”, Federal Emergency Management Agency, Washington, DC

Chopra, A K y R K Goel, (2002). “A modal pushover analysis procedure for estimating seismic demands for buildings”, Earthquake Engineering and Structural Dynamics 31, No. 3, 561-582.

De la Llera, J C y A K Chopra, (1994). “Accidental and natural torsion in earthquake response and design of buildings”, Report No. UCB/EERC-94/07, Earthquake Engineering Research Center, University of California, Berkeley, Cal.

De-la-Colina, J, (1999). "Effects of torsion factors on simple non-linear systems using fully-bidirectional analyses”, Earthquake Engineering and Structural Dynamics 28, 691-706.

De-la-Colina, J, (2003). "Assessment of design recommendations for torsionally unbalanced multistory buildings”, Earthquake Spectra, 19, No. 1, 47-66.

De la Colina, J, (2004). "Programa pushover para edificios a base de marcos”, XIV Congreso Nacional de Ingeniería Estructural, Acapulco, Gro.

Giberson, M, (1969), “Two nonlinear beams with definitions of ductility”, Journal of the Structural Division, Proc. ASCE 95, No. 2, 137-157.

Goel, R K y A K Chopra, (2004). "Evaluation of modal and FEMA pushover analyses: SAC buildings”, Earthquake Spectra 20, No. 1, 225-254.

Humar, J y P Kumar, (1999). "Effect of orthogonal inplane structural elements on inelastic torsional response”, Earthquake Engineering and Structural Dynamics 28, 1071-1097.

International Conference of Building Officials (ICBO), (1997). Uniform Building Code, Whittier, Cal.

Kilar, V y P Fajfar, (1997). Simple push-over analysis of asymmetric buildings, Earthquake Engineering and Structural Dynamics 26, 233-249.

Kim, S y E D’Amore, (1999). “Push-over analysis procedure in earthquake engineering”, Earthquake Spectra 15(3), 417-434. 
Li, K N, (1996). “CANNY-E A general purpose computer program for 3-dimensional nonlinear dynamic analysis of building structures”, Research report, National University of Singapore, Singapur.

Maison, B F, (2005). Discusión del artículo "Evaluation of Modal and FEMA Pushover Analyses: SAC Buildings” por R Goel y A K Chopra,, presentado en Earthquake Spectra, Vol. 20, No. 1 de 2004. Earthquake Spectra 21(1), 275-275.

Moghadam, A S y W K Tso, (1996). "Damage assessment of eccentric multistorey buildings using 3-D pushover analysis”, Proceedings, $11^{\text {th }}$ World Conference on Earthquake Engineering, Acapulco, Gro.

National Research Council of Canada (NRCC), (1995). National Building Code of Canada, Ottawa.

Paret, T F, K K Sasaki, D H Eilbekc y S A Freeman, (1996). “Approximate inelastic procedure to identify failure mechanisms from higher mode effects”, Proceedings, 11th World Conference on Earthquake Engineering, Acapulco, Gro.

Sasaki, K K, S A Freeman y T F Paret, (1998). "Multimode pushover procedure (MMP) - A method to identify the effects of higher modes in a pushover analysis", Proceedings, $6^{\text {th }}$ U.S. National Conference on Earthquake Engineering, Seattle, Washington.

Valdés, J, (2004). “Análisis de efectos sísmicos ortogonales horizontales en terreno blando de la ciudad de México”, Tesis de doctorado, Universidad Nacional Autónoma de México.

Wong, C M y W K Tso, (1995). “Evaluation of seismic torsional provisions in Uniform Building Code”, Journal of Structural Engineering, ASCE, 121(10), 1436-1442. 